Revista Ceuma Perspectivas, Edição Especial.

I Fórum de Meio Ambiente do Estado do Maranhão, Ceuma. Vol. 30, nº1, 2017.

ISSN Eletrônico: 2525-5576.

\title{
RESISTÊNCIA CRUZADA ENTRE AGROTÓXICOS E ANTIFÚNGICOS DE USO CLÍNICO CONTRA Cryptococcus neoformans
}

\section{CROSS-RESISTANCE BETWEEN AGROCHEMICALS AND ANTIFUNGALS FOR CLINICAL USE AGAINST Cryptococcus neoformans}

Ione Cristine da Silva de Araújo ${ }^{1}$; Rayana Larissa Pinheiro Soares Ferreira ${ }^{2}$;

Alessandra Teixeira Macedo ; Joice Castelo Branco Santos ; Julliana Alves Ribeiro dos Santos.

RESUMO: A exposição ambiental aos fungicidas agrícolas abre caminho para a seleção e multiplicação de fungos resistentes a esses compostos. Contudo, este estudo teve como objetivo avaliar a resistência cruzada entre agrotóxicos e antifúngicos clínicos contra Cryptococcus neoformans. Foram determinadas a concentração inibitória mínima (CIM) para os agrotóxicos epoxiconazol e para o carbendazim, e para os antifúngicos fluconazol e anfotericina B pelo teste de microdiluição antifúngica frente a um isolado clínico de Cryptococcus neoformans, antes e após o contato com os fungicidas agrícolas. O fluconazol foi diluído em água, o Epoxiconazol e o Carbendazim foram diluídos em DMSO na concentração de $1500 \mu \mathrm{g} / \mathrm{mL}$. A Anfotericina B foi diluída em DMSO na concentração de $1000 \mu \mathrm{g} / \mathrm{mL}$. As placas foram incubadas a $35^{\circ} \mathrm{C}$ por 72 horas e a CIM foi determinada visualmente como $100 \%$ de inibição do crescimento. Antes do contato com os agrotóxicos, foram observadas altas concentrações inibitórias mínimas para o carbendazim $(64 \mu \mathrm{g} / \mathrm{mL})$, enquanto as CIM da anfotericina B $(2 \mu \mathrm{g} / \mathrm{mL})$, do fluconazol $(0,5 \mu \mathrm{g} / \mathrm{mL})$ e do epoxiconazol $(0,25 \mu \mathrm{g} / \mathrm{mL})$ frente a $C$. neoformans foram menores. No entanto, após o contato com os agrotóxicos, a CIM para fluconazol aumentou 4 vezes $(8 \mu \mathrm{g} / \mathrm{mL})$ e para a anfotericina B aumentou 3 vezes $(16 \mu \mathrm{g} / \mathrm{mL})$. Os resultados do nosso estudo puderam comprovar que há a possibilidade de resistência cruzada entre fungicidas agrícolas e os principais antifúngicos de uso clínico e pode ser uma das hipóteses da resistência primária que vem sendo observada durante o tratamento da criptococose.

PALAVRAS-CHAVE: Cryptococcus; agrotóxicos; resistência; antifúngicos.

\footnotetext{
${ }^{1}$ Discente em Enfermagem da Universidade CEUMA, no período de 2013 a 2017. Bolsista de Iniciação Científica pelo Programa Institucional de Bolsas de Iniciação Científica (PIBIC), atuando no Laboratório de Microbiologia Ambiental (UNICEUMA), inserida nos grupos de pesquisa Mecanismos de modulação de saúde e ambiente no Maranhão e Patogenicidade Microbiana. Email:ionecaraujo@outlook.com

${ }^{2}$ Biomédica, Bacharel em Biomedicina pela Universidade de Ceuma. Mestranda em Biologia Parasitária da Universidade de Ceuma, Bolsista de Iniciação Científica pela Fundação de Amparo à Pesquisa e ao Desenvolvimento Científico e Tecnológico do Maranhão (FAPEMA), no Laboratório de Microbiologia Ambiental (UNICEUMA), inserida no grupo de pesquisa Patogenicidade Microbiana. E-mail:rayana-larissa@hotmail.com

${ }^{3}$ Discente em Biomedicina da Universidade CEUMA, no período de 2016 a 2018. Bolsista de Iniciação Científica pela Fundação de Amparo à Pesquisa e ao Desenvolvimento Científico e Tecnológico do Maranhão (FAPEMA), atuando no Laboratório de Microbiologia Ambiental (UNICEUMA), inserida no grupo de pesquisa Patogenicidade Microbiana. E-mail: alessandra.macedo@hotmail.com

${ }^{4}$ Discente em Biomedicina da Universidade CEUMA, no período de 2016 a 2018. Bolsista de Iniciação Científica pela Coordenação de Aperfeiçoamento de Pessoal de Nível Superior (CAPES), atuando no Laboratório de Prospecção Molecular (UNICEUMA), inserida no grupo de pesquisa Farmacologia de Produtos Naturais. E-mail: joic.cast@ hotmail.com

${ }^{5}$ Graduação em Biomedicina e Mestrado em Biologia de Fungos pela Universidade Federal de Pernambuco, Doutorado em Ciências Biológicas (Microbiologia) pela Universidade Federal de Minas Gerais e Pós-Doutorado (2015-2016) pelo Departamento de Microbiologia da UFMG. Atualmente é Professora e Pesquisadora dos Programas de Mestrado em Biologia Parasitária e do Mestrado em Meio Ambiente da Universidade CEUMA (UNICEUMA). E-mail: julliana.santos@ ceuma.br
} 
ABSTRACT: Environmental exposure to agricultural fungicides opens the way for selection and multiplication of fungi resistant to these compounds. However, this study aimed to evaluate the cross-resistance between pesticides and clinical antifungal drugs against Cryptococcus neoformans. The minimum inhibitory concentration (MIC) for epoxiconazole and carbendazim pesticides and for antifungal fluconazole and amphotericin B were determined by the antifungal microdilution testagainst a clinical isolate of Cryptococcus neoformans before and after contact with agricultural fungicides. Fluconazole was diluted in water, Epoxiconazole and Carbendazim were diluted in DMSO at a concentration of $1500 \mu \mathrm{g} / \mathrm{mL}$. Amphotericin B was diluted in DMSO at the concentration of $1000 \mu \mathrm{g} / \mathrm{ml}$. Plates were incubated at $35^{\circ} \mathrm{C}$ for 72 hours and MIC was determined as $100 \%$ inhibition of growth. The results of the test, prior to contact with pesticides, showed high minimum inhibitory concentrations for Carbendazim (64 $\mu \mathrm{g} / \mathrm{mL}$ ), while Amphotericin B $(2 \mu \mathrm{g} / \mathrm{mL})$, fluconazole $(0.5 \mu \mathrm{g} / \mathrm{mL})$ and epoxiconazole $(0.25 \mu \mathrm{g} / \mathrm{mL})$ compared to $\mathrm{C}$. neoformans were lower. However, after contact with the agrotoxics, the MIC for fluconazole increased 4-fold $(8 \mu \mathrm{g} / \mathrm{mL})$ and for amphotericin B increased 3-fold (16 $\mu \mathrm{g} / \mathrm{mL})$. The results of our study could prove that there is a possibility of cross-resistance between agricultural fungicides and the main antifungal agents of clinical use and may be one of the hypotheses of the primary resistance that has been observed during the treatment of cryptococcosis.

KEY WORDS: Cryptococcus; pesticides; resistance; antifungals.

\section{INTRODUÇÃO}

Desde a antiguidade, o homem usava a terra como fonte de subsistência, ou seja, semeava, lavrava e colhia para manter suas necessidades vitais. Ao longo do tempo, houve a premência de aprimorar e desenvolver sua produção agrícola, pois o cultivo que outrora seria apenas de subsistência a partir desse momento transformou-se em fonte de renda familiar, necessitando industrializar o campo, ou seja, o trabalho que antes era manual passa a ser mecanizado. E entre as mudanças tecnológicas obtidas, foram criados os agrotóxicos, que visavam defender as plantações das pragas (MORO, 2008).

Segundo a Food and Agriculture Organization (FAO), agrotóxico é toda e qualquer substância ou fusão delas que visam prevenir, extinguir ou controlar qualquer praga, que são espécies indesejadas de plantas e animais, causadores de doenças humanas e animais, que propiciam danos durante a produção, processamento, estocagem, transporte ou na distribuição de produtos dealimentícios, agrícolas, madeira e derivados (BRAIBANTE; ZAPPE, 2012).

Desde a Antiguidade clássica agricultores criaram técnicas para combater pragas que se disseminavam pelos cultivos e competiam pelo alimento. Escritos datados na Roma e Grécia já evidenciavam o uso de arsênico e enxofre para o controle de insetos nas plantações. A partir do 
século XVI, na Europa e EUA registram-se o uso de nicotina e piretro, substâncias orgânicas extraídas de plantas (RIGOTTO, 2014). Assim, ao longo do tempo, o homem aperfeiçoou técnicas que controlassem e combatessem as pragas, chegando ao ápice do sucesso na metade do século XX, onde novas técnicas foram estudadas e implementadas no mercado, projetando aumentar substancialmente a produtividade agrícola e resolver o problema da fome nos países em desenvolvimento, esse período foi denominado Revolução Verde, pois se voltava à expansão das agroindústrias, com base na intensiva utilização de sementes híbridas, de insumos industriais (fertilizantes e agrotóxicos), mecanização da produção, uso extensivo de tecnologia no plantio, na irrigação e na colheita, assim como no gerenciamento. Findas as grandes guerras, foi um caminho encontrado pelas indústrias de armamentos para manter os grandes lucros (RIGOTTO, 2014).

O incremento dessas novas tecnologias no campo proporcionou crescimento, em termos de produtividade na agricultura. No entanto, a falta de qualificação da força de trabalho, submeteu comunidades rurais a um conjunto de riscos desconhecidos, originado pelo uso extensivo de um grande número de substâncias químicas perigosas (PERES, MOREIRA, DUBOIS, 2003):

\footnotetext{
O termo agrotóxico inclui inseticidas (controle de insetos), fungicidas (controle de fungos), herbicidas (combate às plantas invasoras), fumigantes (combate às bactérias do solo), algicida (combate a algas), avicidas (combate a aves), nematicidas (combate aos nematoides), moluscicidas (combate aos moluscos), acaricidas (combate aos ácaros), além de reguladores de crescimento, desfoliantes (combate às folhas indesejadas) e dissecantes (BRAIBANTE; ZAPPE, 2012).
}

Inibir o crescimento de fungos constitui-se como uma das finalidades dos agroquímicos. Uma vez que os fungos são patógenos usuais na agricultura, esses microrganismos são organismos ubíquos, saprofíticos e estão presentes em ambientes associados ao solo, plantas e matérias em decomposição. Os principais agentes etiológicos encontrados nesses meios são Aspergillus spp., Cryptococcus spp., Fusarium spp., Fonsecae pedrosoi, Paracoccidioides brasiliensis e fungos dermatófitos (CHAYAKULKEEREE; PERFECT, 2006).

Os agrotóxicos podem exercer alguns efeitos em microrganismos, não necessariamente aqueles para os quais foi destinada à sua aplicação, inclusive os da microbiota do solo. A exposição ambiental a componentes azólicos, que são substâncias químicas utilizadas na medicina para tratamento de infecções fúngicas, e na agricultura como fungicida, podem proporcionar a seleção e multiplicação de fungos resistentes a esses compostos. Assim, trabalhadores rurais ou indivíduos que moram próximo a essas regiões, podem inalar ou ingerir isolados resistentes. Desse modo, 
poderiam ser justificadas muitas infecções causadas por cepas resistentes a tratamento antifúngico com fármacos azólicos (TAKAHASHI; MELHEM, 2014).

Cryptococcus neoformans é uma levedura capsulada, e o agente causador da criptococose, infecção grave que afeta principalmente pacientes imunocomprometidos. Esse fungo caracteriza-se como cosmopolita, pois está presente no meio ambiente, em plantas e animais, além disso, pode ser encontrado em diferentes nichos das áreas ambientais, presume se há interferência direta da utilização de fungicidas azólicos nas lavouras com o desenvolvimento de resistência aos azóis de uso terapêutico (DRUMMOND, 2007). Contudo, este estudo teve como objetivo avaliar a resistência cruzada entre agrotóxicos e antifúngicos clínicos contra Cryptococcus neoformans.

\section{MATERIAIS E MÉTODOS}

\section{PREPARO DAS SOLUÇÕES ESTOQUES DAS DROGAS}

Foram utilizados dois antifúngicos de uso clínico e dois fungicidas agrícolas, os quais foram diluídos em água ou Dimetilsulfóxido (DMSO) de acordo com seu perfil de solubilidade. O fluconazol (SIGMA®)foi diluído em água, o Epoxiconazol(SIGMA®) e o Carbendazim(SIGMA®) foram diluídos em DMSO na concentração de $1500 \mu \mathrm{g} / \mathrm{mL}$. A Anfotericina B(SIGMA®) foi diluída em DMSO na concentração de $1000 \mu \mathrm{g} / \mathrm{mL}$. Todas as drogas após serem diluídas foram filtradas por meio de microfiltros SyeingeFilters - K18 - 230(Kasvi $\left.{ }^{\circledR}\right)$ e estocadas a $4^{\circ} \mathrm{C}$.

\section{MICRORGANISMO E CULTIVO.}

Foi utilizado um isolado clínico de Cryptococcus neoformans pertencente à Coleção de Culturas do Laboratório de Microbiologia Ambiental (cedido gentilmente pelo Laboratório de Micologia da Universidade Federal de Minas Gerais). A amostra de C. neoformans RN01 na primeira fase do experimento foi cultivada em meio Agar Sabouraud, 4\%. O isolado foi ainda cultivado em meio Agar Sabouraud 4\% com adição dos agrotóxicos Carbendazim $(32 \mu \mathrm{g} / \mathrm{mL})$ (Sigma-Aldrich) e Epoxiconazol (0,25 $\mu \mathrm{g} / \mathrm{mL})$ (Sigma-Aldrich). As placas foram incubadas a $35^{\circ} \mathrm{C}$ durante 72 h. Em seguida, foi determinada a concentração inibitória mínima (CIM) do isolado de $C$. neoformans, antes e após o contato com os agrotóxicos. Para realização dos testes da CIM foi preparada uma suspensão do fungo em solução salina $(\mathrm{NaCl} 0,85 \%)$ e ajustado para 0,5 da escala de Mc Farland $=10^{6}$ células/mL. Foi realizada uma diluição 1:50 em RPMI e, logo em seguida, uma 
diluição de 1:20, para alcançar a concentração de $10^{3} \mathrm{UFC} / \mathrm{mL}$ que foi a concentração utilizada para o teste.

\section{CONCENTRAÇÃO INIBITÓRIA MÍNIMA (CIM)}

Foi realizada a determinação da concentração inibitória mínima dos agrotóxicos epoxiconazol, carbendazim e dos antifúngicos clínicos: fluconazol (Sigma-Aldrich) e anfotericina B (Sigma-Aldrich) frente ao isolado clínico Cryptococcus neoformans RN01 antes e após a exposição aos agrotóxicos por meio do teste de microdiluição em placa (CLSI, 2008), como demonstrado na Figura 1. A partir das soluções estoque já preparadas, foram feitas diluições seriadas em Meio RPMI para alcançar as faixas de concentrações $0,25 \mu \mathrm{g} / \mathrm{mL}-128 \mu \mathrm{g} / \mathrm{mL}$ (para o fluconazol, epoxiconazol e carbendazim) e $0,03 \mu \mathrm{g} / \mathrm{mL}-16 \mu \mathrm{g} / \mathrm{mL}$ (para a anfotericina B) e adicionado o inóculo de $10^{3}$ células $/ \mathrm{mL}$ nas placas. As placas foram incubadas a $35^{\circ} \mathrm{C}$ durante 72 horas e a CIM foi determinada visualmente como $100 \%$ de inibição do crescimento.

Figura 1: Divisão da plana de Microdiluição do teste de Concentração Inibitória Mínima (CIM).

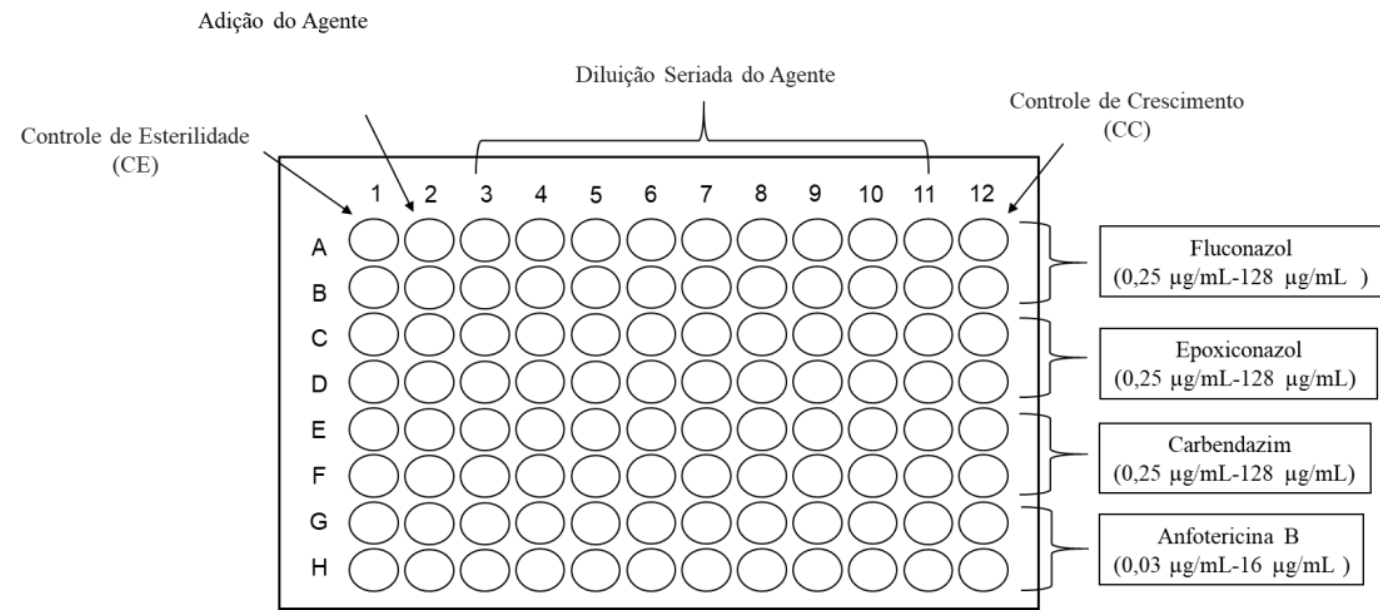

\section{RESULTADOS E DISCUSSÃO}

FONTE: Autores.

O resultado do primeiro teste de Concentração Inibitória Mínima (CIM) com o isolado clínico Cryptococcus neoformans RN01 ainda sem contato com os agrotóxicos representados na Tabela 1 foram: fluconazol $(0,5 \mu \mathrm{g} / \mathrm{mL})$ e anfotericina $\mathrm{B}(2 \mu \mathrm{g} / \mathrm{mL})$ e para o agrotóxico epoxiconazol $(0,25 \mu \mathrm{g} / \mathrm{mL})$, já para o carbendazim, observou-se uma concentração inibitória mínima mais elevada com um valor de CIM igual a $32 \mu \mathrm{g} / \mathrm{mL}$. 
Tabela 1. Resultados dos testes de CIM com a estirpe de $C$. neoformans RN01 antes do contato com os Agrotóxicos.

\begin{tabular}{lc}
\hline $\begin{array}{c}\text { Antifúngicos e } \\
\text { Agrotóxicos }\end{array}$ & $\begin{array}{c}\text { ConcentraçãoInibitória Mínima } \\
\text { (CIM) do Isolado Clinico C. neoformans RN01 }\end{array}$ \\
\hline $\begin{array}{c}\text { Fluconazol } \\
\text { (Antifúngico) }\end{array}$ & $0,5 \mu \mathrm{g} / \mathrm{mL}$ \\
$\begin{array}{c}\text { Epoxiconazol } \\
\text { (agrotóxicos) }\end{array}$ & $0,25 \mu \mathrm{g} / \mathrm{mL}$ \\
$\begin{array}{c}\text { Carbendazim } \\
\text { (agrotóxico) } \\
\text { Anfotericina B } \\
\text { (antifúngico) }\end{array}$ & $32 \mu \mathrm{g} / \mathrm{mL}$ \\
\hline
\end{tabular}

Fonte: Autores.

Ao analisar o resultado do segundo teste, em que a concentração inibitória mínima foi determinada antes e após o contato com os agrotóxicos, observou-se que os resultados de CIM tantos para os agrotóxicos como para os antifúngicos aumentaram (Tabela 2). Quando a amostra foi cultivada no meio Agar Sabouraud com o agrotóxico Carbendazim, os resultados de concentração inibitória mínima dos fungicidas agrícolas aumentaram $2 \mathrm{x}$ tendo como valor de concentração de $0,5 \mu \mathrm{g} / \mathrm{mL}$, para o Epoxiconazol e $64 \mu \mathrm{g} / \mathrm{mL}$ para o Carbendazim, já o fluconazol teve seu valor de CIM aumentado 4 x $(8 \mu \mathrm{g} / \mathrm{mL})$, e a anfotericina B aumentou 3x $(16 \mu \mathrm{g} / \mathrm{mL})$.

Submetendo o isolado clínico de C. neoformans ao meio de cultivo com o agrotóxico Epoxiconazol, o próprio epoxiconazol demonstrou um perfil de resistência maior, elevando sua CIM 4x mais resultando em um valor de $4 \mu \mathrm{g} / \mathrm{mL}$, já o carbendazim aumentou 2x $(64 \mu \mathrm{g} / \mathrm{mL})$, o fluconazol aumentou para 4 vezes a sua concentração $(8 \mu \mathrm{g} / \mathrm{mL})$, e a anfotericina B teve seu valor de CIM aumentado 3 vezes $(16 \mu \mathrm{g} / \mathrm{mL})$. 
Tabela 2. Resultados dos testes de CIM com a estirpe de $C$. neoformans RN01 após odo contato com os Agrotóxicos.

\begin{tabular}{|c|c|c|}
\hline \multirow{2}{*}{$\begin{array}{l}\text { Antifúngicos e } \\
\text { Agrotóxicos }\end{array}$} & \multicolumn{2}{|c|}{$\begin{array}{l}\text { Concentração Inibitória Mínima (CIM) do isolado clinico de } \\
\text { C. neoformans RN01 após o contato com os Agrotóxicos }\end{array}$} \\
\hline & Epoxiconazol & Carbendazim \\
\hline $\begin{array}{l}\text { Fluconazol } \\
\text { (Antifúngico) }\end{array}$ & $8 \mu \mathrm{g} / \mathrm{mL}$ & $8 \mu \mathrm{g} / \mathrm{mL}$ \\
\hline $\begin{array}{l}\text { Epoxiconazol } \\
\text { (agrotóxicos) }\end{array}$ & $4 \mu \mathrm{g} / \mathrm{mL}$ & $0,5 \mu \mathrm{g} / \mathrm{mL}$ \\
\hline $\begin{array}{l}\text { Carbendazim } \\
\text { (agrotóxico) }\end{array}$ & $64 \mu \mathrm{g} / \mathrm{mL}$ & $64 \mu \mathrm{g} / \mathrm{mL}$ \\
\hline $\begin{array}{l}\text { Anfotericina B } \\
\text { (antifúngico) }\end{array}$ & $16 \mu \mathrm{g} / \mathrm{mL}$ & $16 \mu \mathrm{g} / \mathrm{mL}$ \\
\hline
\end{tabular}

Fonte: Autores.

A resistência aos azólicos clínicos está cada vez mais relacionada ao fracasso no tratamento do paciente (HOWARD et. al., 2009). O uso excessivo dos azólicos na agricultura como os fungicidas inibidores de desmetilase (DMI), não só influenciou os fungos patogênicos para as plantas, mas também aqueles de espécies saprófitas, tais como: Histoplasma, Aspergillus, Coccidioides e o Cryptococcus que é o alvo deste estudo (BASTOS RW et al, 2017). Assim, elevando também a frequência de isolados resistentes aos múltiplos triazólicos (MTR). Na agricultura, azólicos são comercializados para o controle e a proteção de plantas contra os agentes fúngicos que causam perdas na colheita. Estes sendo o principal tratamento para doenças humanas e agrícolas causadas pelos fungos, sendo de extrema importância a preocupação entre as espécies fúngicas e os fungicidas que possuem mecanismos de ação semelhantes aos antifúngicos utilizados em humanos (HOF,2001).

De acordo com o estudo de Faria-Ramos I e colaboradores (2014), os valores de CIM para Aspergillus fumigatus a azólicos após a exposição ao procloraz (que também é um azólico agrícola), observou-se um aumento da CIM para o Voriconazol, Posaconazol e Itraconazol. Este estudo corrobora com Meletiadis J (2012), onde observou-se a resistência cruzada entre azólicos clínicos quando todos os isolados passaram de sensíveis para resistentes, após o contato com agroquímicos. Esse estudo evidenciou que a inclusão de um antifúngico agrícola ampliou o surgimento de resistência cruzada a exposição aos azólicos clínicos.

Da mesma maneira, Müller (2007) relatou a resistência cruzada entre azólicos e isolados de leveduras da orofaringe de pacientes imunocomprometidos e de isolados ambientais contra azólicos clínicos e agrícolas. 


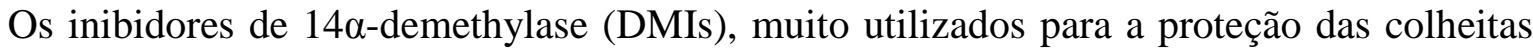
podem estar correlacionados à resistência aos antifúngicos clínicos. A maioria das DMIs associa a sua estrutura aos triazólicos médicos, resultando em ligações idênticas e elevado nível de resistência cruzada (EVELINE; SNELDRS, 2012).

O nosso estudo reforça que o uso indiscriminado de antifúngicos azólicos na natureza pode causar graves riscos à saúde humana, consequentes reações cruzadas com antimicrobianos de uso clínico. São necessários novos estudos para compreender os mecanismos de resistência cruzada e para estimular a pesquisa de alternativas mais seguras para o uso na agricultura.

\section{CONCLUSÃO}

A criptococose vem se tornando um problema de saúde pública pela ocorrência de casos novos que está aumentando do decorrer dos tempos, tendo como uma preocupação ainda maior o número de pacientes que estão apresentando uma resistência aos antifúngicos utilizados para o tratamento desta patologia.

Os resultados do nosso estudo puderam comprovar que há a possibilidade de resistência cruzada entre fungicidas agrícolas e os principais antifúngicos de uso clínico e pode ser uma das hipóteses da resistência primária que vem sendo observada durante o tratamento da criptococose.

Este estudo vem com intuito de encorajar novos estudos sobre o tema em questão e tentar conscientizar os leitores que o uso excessivo dos fungicidas agrícolas pode acarretar em uma mutação aos fungos e a outros microrganismos, causando uma resistência dos pacientes aos tratamentos. Os questionamentos acerca da resistência adquirida pelos microrganismos, desperta o interesse para a busca de novas maneiras para combater as pragas existentes no solo e nas plantações agrícolas.

\section{REFERÊNCIAS}

BASTOS RW, CARNEIRO HCS, DE OLIVEIRA LVN, ROCHA KM, FREITAS GJC, COSTA MC, MAGALHÃES TFF, DE CARVALHO VSD, ROCHA CE, FERREIRA GF, DE PAIXÃO TA, MOYRAND F, JANBON G, SANTOS DA Environmental triazole induces cross-resistance to clinical drugs and affects morphophysiology and virulence of cryptococcus gattii and $c$. neoformans. Antimicrobial Agents and Chemotherapy, 2017.

BRAIBANTE, M. E. F.; ZAPPE, J. A. A química dos agrotóxicos. Química nova na escola, 2012. 
DRUMMOND, Elislene Dias et al .Comportamento de amostras ambientais e clínicas de Cryptococcus neoformans frente a fungicidas de uso agronômico e ao fluconazol. Revista da Sociedade Brasileira de Medicina Tropical. Uberaba, v. 40, n. 2, p. 209-211, 2007

FARIA-RAMOS I, FARINHA S, NEVES-MAIA J, TAVARES RIBEIRO P, MIRANDA M I, ESTEVINHO M L, VAZ-PINA C, RODRIGUES G A, Development of cross-resistance by Aspergillus fumigatus to clinical azoles following exposure to prochloraz, an agricultural azole. BMC Microbiology,2014.

HOWARD SJ, CERAR D, ANDERSON MJ, ALBARRAG A, FISHER MC, PASQUALOTTO AC, LAVERDIERE M,ARENDRUP MC, PERLIN DS, DENNING DW. Freqüência e evolução da resistência de Azole em Aspergillusfumigatus associada à falha do tratamento. Emergência Infect Dis, 2009.

HOF H . Critical annotations to the use of azole antifungals for plant protection. Antimicrob Agents Chemother, 2001.

MELETIADIS J, MAVRIDOU E, MELCHERS WJ, MOUTON JW, VERWEIJ PE. Valores de corte epidemiológico para azoles e Aspergillusfumigatus com base em uma nova abordagem matemática incorporando análise de seqüência cyp51A. AgentesantimicrobianosChemother. 2012.

MORO, B. P. Um estudo sobre a utilização de agrotóxicos e seus riscos na produção do fumo no município de Jacinto Machado/SC. Universidade do Extremo Sul Catarinense - UNESC, 2008.

MÜLLER, F.-M. C., A. STAUDIGEL, S. SALVENMOSER, A. TREDUP, R. MILTENBERGER, AND J. V.HERRMANN. Cross-resistance to medical and agricultural azole drugs in yeasts from theoropharynx of human immunodeficiency virus patients and from environmental Bavarian vine grapes.Antimicrob. Agents Chemother, 2007.

PERES, F.; MOREIRA, J. C.; DUBOIS, G. S. Agrotóxicos, saúde e ambiente: uma introdução ao tema. Rio de Janeiro: Editora FIOCRUZ, 2003.

RIGOTTO, R.M. Agrotóxicos. Núcleo TRAMAS- Trabalho, Meio Ambiente e Saúde para a Sustentabilidade Universidade Federal do Ceará, 2014.

SNELDERS, E.; CAMPOS, M. T. S.; KARAWAJCZYK, A.; SCHAFTENAAR, G.;KEMA, G. H. J.; LEE, H. A. V. D.; KLAASSEN, C. H.; MELCHERS, W. J. G.; VERWEIJ, P. E. Triazole Fungicides Can Induce Cross-Resistance to Medical Triazoles in Aspergillus fumigatus. PLOS, 2012.

TAKAHASHI, J. P., MELHEM, M. de S. C. Uso de fungicidas na agricultura e resistência a antifúngicos na clínica médica. BolInst Adolfo Lutz. 2014. 